\title{
Backstepping Control of a Rigid Body
}

\author{
S. Torkel Glad and Ola Härkegård ${ }^{1}$
}

\begin{abstract}
A method for backstepping control of rigid body motion is proposed. The control variables are torques and the force along the axis of motion. The proposed control law and lyapunov function guarantee asymptotic stability from all initial values except one singular point.
\end{abstract}

\section{Introduction}

An important tool for nonlinear control synthesis is backstepping, see e.g. [4], [8]. The idea is to extend a Lyapunov function from a simple system to systems involving additional state variables and at the same time design the feedback control to guarantee stability. This technique has been successful in several applications, [1, 2, 3, 9]. Recently backstepping design has been successfully applied to the control of aircraft, $[5,6,7]$. The aircraft dynamics is essentially described by rigid body dynamics in combination with equations describing the aerodynamic forces. There are several ways of designing controllers for rigid body equations occuring in various applications, see e. g. $[10,11]$. The purpose of the present paper is to formulate a design method for a controlled rigid body using backstepping techniques. The design can then be specialized to aircraft control problems or the control of various types of vehicles.

\section{Rigid body dynamics}

We assume that the controlled object is a rigid body with mass $m$. We describe the motion in a body fixed coordinate system with the origin at the centre of mass and obtain the equations:

$$
\begin{aligned}
\dot{V} & =-\omega \times V+\frac{1}{m} F \\
I \dot{\omega} & =-\omega \times I \omega+M
\end{aligned}
$$

where $V$ is the velocity, $\omega$ is the angular velocity, $F$ is the external force and $M$ is the external torque (all these quantities are vectors with three components). I is the moment of inertia. We will assume that the force

\footnotetext{
${ }^{1}$ Dept. of Electrical Engineering, Linköpings universitet, SE58183 Linköping, Sweden. torkel@isy.liu.se, ola@isy.liu.se
}

has the form

$$
F=m\left(F_{a}(V)+u_{v} \hat{V}\right)
$$

where $\hat{V}=\frac{1}{|V|} V$ and $u_{v}$ is a control variable. The first part, $F_{a}$, corresponds to aerodynamic or hydrodynamic forces, and the second part models approximately the thrust action of an engine aligned with the velocity vector. The moment $M$ is assumed to depend on $V, \omega$ and control variables.

\section{Stationary motion}

Consider a motion with $V=V_{o}, \omega=\omega_{o}$ where $V_{o}, \omega_{o}$ are constants. The velocity equation is then

$$
\omega_{o} \times V_{o}=F_{a}\left(V_{o}\right)+u_{v} \hat{V}_{o}
$$

where $\hat{V}_{o}=\frac{1}{\left|\hat{V}_{o}\right|} V_{o}$. Multiplying with $\hat{V}_{o}$ shows that $u_{v}$ has to satisfy

$$
u_{v}=-\hat{V}_{o}^{T} F_{a}\left(V_{o}\right)
$$

Then $\omega_{o}$ can be calculated from

$$
\omega_{o}=\frac{1}{\left|V_{o}\right|}\left(\hat{V}_{o} \times F_{a}\left(V_{o}\right)\right)+\gamma \hat{V}_{o}
$$

where $\gamma$ is an arbitrary constant. If $u_{v}$ and $M$ can be chosen arbitrarily it is thus possible to achieve a stationary motion for any value of $V_{o}$.

\section{Backstepping design}

In this section we develop a backstepping design to make $V_{o}, \omega_{o}$ a stable equilibrium. Define

$$
u_{M}=I^{-1}(M-\omega \times I \omega)
$$

We will regard $u_{M}$ as the control signal. Then the dynamics is given by

$$
\begin{aligned}
\dot{V} & =-\omega \times V+F_{a}(V)+u_{v} \hat{V} \\
\dot{\omega} & =u_{M}
\end{aligned}
$$

First regard the angular velocity $\omega$ (together with $u_{v}$ ) as the control variable. Let $V_{0}$ be the desired velocity vector and introduce the Lyapunov candidate

$$
W_{1}=\frac{1}{2}\left(V-V_{o}\right)^{T}\left(V-V_{o}\right)
$$


Choose a control of the form

$$
\omega=\omega^{\mathrm{des}}=\bar{\omega}+\frac{1}{|V|^{2}} V \times F_{a}(V)
$$

After some manipulations this gives

$$
\dot{W}_{1}=-\bar{\omega}^{T}\left(V_{o} \times V\right)+\bar{u}_{v}\left(V-V_{o}\right)^{T} \hat{V}
$$

where $u_{v}=\bar{u}_{v}-\hat{V}^{T} F_{a}$. Choosing

$$
\bar{\omega}=k_{1}\left(V_{o} \times V\right), \quad \bar{u}_{v}=-\left(V-V_{o}\right)^{T} \hat{V}
$$

then gives

$$
\begin{gathered}
\omega^{\text {des }}=k_{1}\left(V_{o} \times V\right)+\frac{1}{|V|^{2}} V \times F_{a}(V) \\
\left.\dot{W}_{1}\right|_{\omega=\omega^{\text {des }}}=-k_{1}\left|V_{0} \times V\right|^{2}-\left(\left(V-V_{o}\right)^{T} \hat{V}\right)^{2} \leq 0
\end{gathered}
$$

In this expression $\left.\dot{W}_{1}\right|_{\omega=\omega^{\mathrm{des}}}=0$ only if $V=V_{o}$ (provided the singularity $V=0$ is avoided, which can be done e.g. by starting so that $\left.\left|V-V_{o}\right|<\left|V_{o}\right|\right)$. The lyapunov function thus guarantees convergence to the desired $V=V_{o}$.

Define

$$
\xi=\omega-\omega^{\mathrm{des}}
$$

In the new variables the dynamics is

$$
\begin{aligned}
\dot{V} & =-\xi \times V+k_{1}\left(|V|^{2} V_{0}-\left(V^{T} V_{0}\right) V\right)+\bar{u}_{v} \hat{V} \\
\dot{\xi} & =u_{M}+\phi(V, \xi)
\end{aligned}
$$

where $\phi(V, \xi)=\frac{d}{d t}\left(\omega^{\text {des }}\right)$. Introducing

$$
W_{2}=k_{2} W_{1}+\frac{1}{2} \xi^{T} \xi
$$

gives

$\dot{W}_{2}=-k_{1} k_{2}\left|V_{0} \times V\right|^{2}-k_{2}\left(\left(V-V_{o}\right)^{T} \hat{V}\right)^{2}-k_{3} \xi^{T} \xi \leq 0$

if we select the control

$$
u=k_{2} V_{0} \times V-\phi-k_{3} \xi
$$

Since $\dot{W}_{2}=0$ only occurs for $V=V_{o}, \xi=0$ (except for the singular case $V=0$, discussed above) there will be convergence to $V=V_{o}, \xi=0$.

\section{Conclusions}

We have proposed a control law that steers the velocity and angular velocity vectors to desired values. The control law uses external torques and a force along the velocity vector. This configuration is similar to, but not precisely equal to the one used in aircraft control, where control surfaces generate torques and the engine gives a longitudinal force. However, our proposed rigid body control could inspire new aircraft control designs. An interesting extension would be to take the orientation into account, which would make it possible to e.g. include the effect of forces like gravity.

Acknowledgement This work was supported by the Swedish Research Council (Vetenskapsrådet).

\section{References}

[1] M. T. Alrifai, J. H. Chow, and D. A. Torrey. A backstepping nonlinear control approach to switched reluctance motors. In Proc. of the 37th IEEE Conference on Decision and Control, pages 4652-4657, Dec. 1998.

[2] J. J. Carroll, M. Schneider, and D. M. Dawson. Integrator backstepping techniques for the tracking control of permanent magnet brush DC motors. In Conference Record of the 1993 IEEE Industry Applications Society Annual Meeting, pages 663-671, Oct. 1993.

[3] T. I. Fossen and Å. Grøvlen. Nonlinear output feedback control of dynamically positioned ships using vectorial observer backstepping. IEEE Transactions on Control Systems Technology, 6(1):121-128, Jan. 1998.

[4] R. A. Freeman and P. V. Kokotović. Robust Nonlinear Control Design: State-Space and Lyapunov Techniques. Birkhäuser, 1996.

[5] O. Härkegård. Flight control design using backstepping. Licentiate thesis 875, Department of Electrical Engineering, Linköpings universitet, Mar. 2001.

[6] O. Härkegård and S. T. Glad. A backstepping design for flight path angle control. In Proc. of the 39th Conference on Decision and Control, pages 3570-3575, Sydney, Australia, Dec. 2000.

[7] O. Härkegård and S. T. Glad. Flight control design using backstepping. In Proc. of the IFAC NOLCOS'01, St. Petersburg, Russia, July 2001.

[8] M. Krstić, I. Kanellakopoulos, and P. Kokotović. Nonlinear and Adaptive Control Design. John Wiley \& Sons, 1995.

[9] M. Krstić and P. V. Kokotović. Lean backstepping design for a jet engine compressor model. In Proc. of the 4th IEEE Conference on Control Applications, pages 10471052, 1995.

[10] C. A. Woolsey, A. M. Bloch, N. E. Leonard, and J. E. Marsden. Dissipation and controlled euler-poincare systems. In Proceedings of the 40th IEEE Conference on Decision and Control, pages 3378-3383, Orlando, Florida, December 2001.

[11] C. A. Woolsey and N. E. Leonard. Global asymptotic stabilization of an underwater vehicle using internal rotors. In Proceedings of the 38th IEEE Conference on Decision and Control, pages 2527-2532, Phoenix, Arizona, December 1999. 